\title{
A check-list of Finnish Myxomycetes
}

\author{
MARJA HÄRKÖNEN
}

\begin{abstract}
HÄRKÖNEN, M. 1979: A check-list of Finnish Myxomycetes. - Karstenia 19: $8-18$.

Finnish research on Myxomycetes is briefly reviewed, and an annotated check-list is given of all the names by which the Myxomycetes are listed in the Finnish literature, with references to the biological provices where the species have been found. Special attention is paid to the identity of the species described by P.A.Karsten and W.Nylander from Finland. Diderma simplex (Schroeter) G.Lister is reported as new to Finland.

At present the Finnish herbaria contain material of 132 species of Myxomycetes from Finland. Fourteen further species are mentioned in the literature, but no proper specimens could be traced.
\end{abstract}

Marja Härkönen, Department of Botany, University of Helsinki, Unioninkatu 44, SF00170 Helsinki 17, Finland

The first reliable records of myxomycete species in Finland are evidently those in Wahlenberg's Flora Lapponica (Wahlenberg 1812), where Lycogala miniata $(=L$. epidendrum (L.) Fries) is reported from Sodankylä and Stemonitis fasciculata $(=S$. fusca Roth) from Inari, both in northern Finland. Before that, however, attention had been paid to Fuligo septica (L.) Wiggers, which even plays some part in Finnish mythology (see Forsman 1898), and is known by such Finnish names as: paran voi ('troll's butter'), paran paska ('troll's shit'), paran oksennus ('troll's vomit') (Reinholm 1851, Suhonen 1936). This peculiar creature had many local names in ancient Scandinavia as: trollsmör, bäresmör, svamp $i$ hus, päuksmöjr, bårådrit in Sweden and troldkaeringsmör in Norway (Fries 1880, Jenssen-Tusch 1867). Only two other species of Myxomycetes have received Finnish names: Physarum album - valkoinen limasieni (see Mela 1892) and Lycogala epidendrum - sudenmaito (see Ulvinen in Mossberg et al. 1977).

Mycofloristical investigation in Finland was started by William Nylander (see Norrlin 1899, Hintikka 1950). After having visited Elias Fries for several weeks in Upsala, he collected fungi in Finland in 1851-1852, and his specimens are the oldest in the fungus collections of the Botanical Museum of the University of Helsinki. Nylander also reported some species of Myxomycetes and even described two new taxa (Nylander 1859, Nylander \& Saelan 1859), both of which have later been included in synonym lists of other species.

P.A.Karsten, the famous early Finnish mycologist (see Hintikka 1935, 1950) devoted the whole of his scientific activity, lasting over 50 years, to floristic and taxonomic mycology, leaving behind him a large collection, mostly from Etelä-Häme and VarsinaisSuomi. His records of Myxomycetes from Finland are found in four exsiccati collections and nine publications. The first of the latter concerned fungi collected during his fruitful trip to eastern Lapland in 1861. Among the 425 species of fungi, were 18 species of Myxomycetes, some of which were collected from the Kola Peninsula, in NW Russia (Karsten 1966a). His main work 'Mycologia Fennica', comprising over 1000 pages, contains descriptions of 1662 species of fungi, among them 82 Myxomycetes (Karsten 1879). Karsten presented several new taxa and combinations of Myxomycetes, but none of them was treated as valid in Martin \& Alexopoulos (1969), though this was not completely justified in every case (see Härkönen 1979).

T.J.Hintikka made the first revision of Finnish Myxomycetes (Hintikka 1920), listing 91 species. This publication is regarded as a considerable achievement, and his most important mycofloristical work (see Suomalainen 1948, Kujala 1953, 1954, Kotilainen 1954). Some years later he prepared an exsiccati collection of 20 species of Myxomycetes (Hintikka 1924), but after that his attention was directed to other fields, mostly plant pathology and the history of botany. He went on collecting slime moulds, however, mainly in Uusimaa and PohjoisSavo, but never found time to name his specimens.

In the following 50 years, only a few short lists of Finnish Myxomycetes were published (Eklund 1943, V.Hintikka 1963, Kallio \& Kankainen 1966), and 
although the collections of the botanical museums in Finland (H, HFR, HPP (= H-LA), KUO, OULU, TUR) received many specimens of Myxomycetes, they were identified in only a few cases (see Marklund 1954, Roivainen 1961, 1962, 1963, 1964, Kukkonen \& Ahti 1978). In 1965 I began the systematic identifications of this material (see Kallio \& Kankainen 1966, Härkönen 1974 (unfortunately with many misprints and an incorrect way of indicating the authors), 1979). Including the species found by me (Härkönen 1974, 1977a,b, 1978a,d, 1979, Härkönen \& Koponen 1978, Ulvinen et al. 1979), the number of myxomycete species in the Finnish herbaria is now 132. Fourteen further species are mentioned in the literature, but no proper specimens could be found for them.

The picture of the distribution of myxomycete species in Finland is still far from complete. Collecting has been rather random and most active in certain biological provinces (main collectors in brackets): Varsinais-Suomi (Karsten), Uusimaa (many collectors), Satakunta (Laurila, Härkönen), Etelä-Häme (Karsten), Pohjois-Savo (T.J.Hintikka), Pohjois-Pohjanmaa (Ohenoja, Ulvinen), Kuusamo (Laurila, Härkönen), Inarin Lappi (Härkönen). The most common myxomycete species in Finland seem to be: Didymium melanospermum, Fuligo septica, Leocarpus fragilis, Lycogala epidendrum, Mucilago crustacea, Physarum nutans, Stemonitis axifera, $S$. fusca, Trichia decipiens and $T$. varia.

In this paper the studies on Finnish Myxomycetes are summarized in the form of a check-list. The list of references (p. 16) gives the publications concerning them, except course books and encyclopedias. The publications containing chorological or taxonomical information are preceded by a serial number. The many foreign monographs on slime moulds that mention Finland in the notes about the distribution are not listed here, because they seem to refer to earlier Finnish studies and so do not add anything to the current konwledge. The only exception is Lister (1925) (see note 3).

In the check-list all the names used for Myxomycetes in the Finnish literature are given in alphabetical order. The numbers after the names refer to the publications in which they appear. The nomenclature is mostly according to Martin \& Alexopoulos (1969) or Nannenga-Bremekamp (1974): if it is not, the references are given after the name. The names regarded as valid are printed in boldface, the synonyms in ordinary type. If the Finnish herbaria ( $\mathrm{H}, \mathrm{HFR}$, HPP ( = H-LA), KUO, OULU, TUR) no longer contain any specimens of the species, or the specimen is in a very poor condition, the name is given in brackets. The taxa or references erroneously reported from Finland are given in square brackets. For each species there is a list of the biological provinces where it has been collected, compiled from the literature or specimens in the Finnish herbaria. The abbreviations used for the biological provinces are explained in Fig.1. A separate set of notes is appended to the check-list, and these include discussions of the names given by P.A.Karsten and W.Nylander.

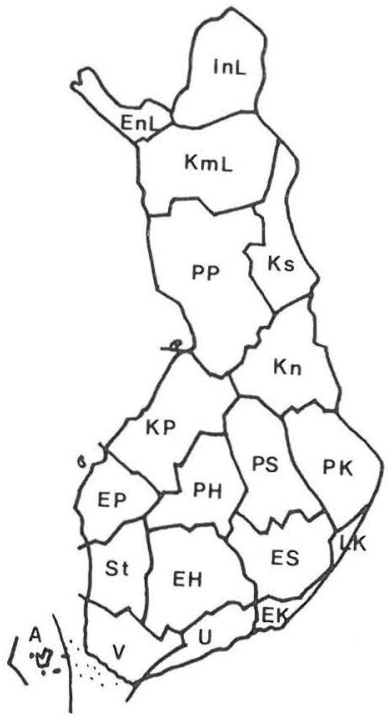

Fig. 1. The abbreviations for the biological provinces in Finland. 


\section{The check-list}

Aethalium septicum $\rightarrow$ Fuligo septica

A. septicum var. violaceum $\rightarrow$ Fuligo septica

A maurochaete atra (Albert. \& Schweinitz)Rostaf. 24, A. fuliginosa $3,5,6$, Reticularia atra 32 . - V, U, St, EH, EP, PS.

A. fuliginosa $\rightarrow$ A. atra

Angioridium sinuosum $\rightarrow$ Physarum bivalve

Arcyodes incarnata (Albert. \& Schweinitz) O.F. Cook, Cornuvia anomala 24, Lachnobolus congestus 3, 6, Trichia anomala 22. - V, EK, St, EH. Note 1.

Arcyria adnata $\rightarrow$ A. incarnata

A. cinerea (Bull.) Pers. 3, 6, 7, 9, 22, 24. - V, U, St, EH, PS, PK, KmL, EnL, InL.

A. denudata (L.) Wettst. 3, 6, A. punicea [19 is A. incarnata] 22, 24. - V, U, St, EH, PS, PP, InL.

A. ferruginea Sauter 3, 6, 24. - V, U, EK, St, Kn, PP.

A. incarnata (Pers.) Pers. 2, 3, 4, 5, 6, 7, 16, 19 (as 'A. punicea'), 22, 38, A. adnata 24. - V, U, EK, St, EH, EP, PS, PP, Ks, InL.

A. nutans $\rightarrow$ A. obvelata

A. obvelata (Oeder) Onsberg, Mycologia 70: 1286 (1978). A. nutans 3, 4, 5, 6, 22, 24, 37, 38. - A, V, U, EK, St, EH, PS, KP, PP, Ks, InL. Note 2.

(A. oerstedtii Rostaf. 3, 6, EH.)

A. pomiformis (Leers) Rostaf. 3, 6, 7, 16, 24. - V, U, EH, PS, PP, InL.

A. punicea $\rightarrow$ A. denudata

(A. stipata (Schweinitz) A. Lister 3, 6,) Note 3.

Badhamia capsulifera (Bull.) Berk. 3, 6, [30 is B. utricularis], B. hyalina 24. - ES.

B. decipiens $\rightarrow$ Physarum decipiens

B. foliicola A. Lister 3, 6, 13. - U, EK.

B. hyalina $\rightarrow$ B. capsulifera

B. lilacina (Fries) Rostaf. 13. - V. Note 4.

B. utricularis (Bull.) Berk. 3, 6, 24, 30, (as 'B capsulifera'), B. utricularis var. melaleuca 23, 24, Physarum melaleucum 32, P. utriculare 22, 31, Physarum utriculare var. melaleucum 31. - A, V, U, EK, St, EH, EP, PS, PP, InL. Note 5.

B. utricularis var. melaleuca $\rightarrow$ B. utricularis

Brefeldia maxima (Fries) Rostaf. 3, 6, 24. - EH.

Calomyxa metallica (Berk.) Nieuwl. 7, Margarita metallica 3, 6, Oligonema aeneum 24. - V, U, EH. Note 6.

Carcerina valvata $\rightarrow$ Physarum bivalve

Ceratiomyxa fruticulosa (O.F. Müller) Macbr. 2, 3, 5, 6, 38, C. fruticulosa var. porioides 3, Ceratium aureum 27, C. hydnoides 17, 23, 28, C. porioides 23, 28. - V, U, EK, St, EH, PS, PK, PP, Ks, Inl.

C. fruticulosa var. porioides $\rightarrow$ C. fruticulosa
Ceratium aureum $\rightarrow$ Ceratiomyxa fruticulosa

C. hydnoides $\rightarrow$ Ceratiomyxa fruticulosa

C. porioides $\rightarrow$ Ceratiomyxa fruticulosa

Chondrioderma difforme $\rightarrow$ Didymium difforme

C. michelii $\rightarrow$ Diderma hemisphaericum

C. radiatum $\rightarrow$ Diderma radiatum

C. testaceum $\rightarrow$ Diderma testaceum

Comatricha alta Preuss 13. - U.

C. elegans (Racib.) G. Lister 7. - U.

C. ellae Härkönen, Karstenia 18: 23(1978) 11, C. nannengae 8. - U. Note 7.

C. friesiana $\rightarrow$ C. nigra

[C. irregularis Rex 6 (see Symphytocarpus amaurochaetoides)]

C. Iaxa Rostaf. 6. - U, PK, EnL.

C. nannengae $\rightarrow$ C. ellae

C. nigra (Pers.) Schroeter 3, 5, 6, 7, 9, 16, 37, 38, C. friesiana 24, Stemonitis friesiana 19, 22, S. ovata 22. - A, V, U, St, EH, PS, PP, Ks, KmL, EnL, InL.

C. typhina $\rightarrow$ Stemonitopsis typhina

C. typhoides $\rightarrow$ Stemonitopsis typhina

Cornuvia anomala $\rightarrow$ Arcyodes incarnata

Craterium leucocephalum (Pers.) Ditmar 3, 6, - V, U, EK, PS, PP.

C. minutum (Leers) Fries 3, 6, 22, 24, C. pedunculatum 24. - V, EH, PS.

C. pedunculatum $\rightarrow$ C. minutum

Cribraria argillacea (Pers.) Pers. 3, 6, 22, 24, 38. - V, U, EK, St, EH, EP, PS, PK, KP, Ks.

[C. aurantiaca (Schrader 3, 6 are C. vulgaris 24.] Note 8 .

C. cancellata (Batsch) Nann.-Brem., Dictydium cancellatum $3,4,6$, D. cernuum 24 , D. umbilicatum 22. - V, U, St, EH, EP, PS, PK.

C. intricata Schrader 3, 6. - V.

(C. microcarpa (Schrader) Pers. 3, 6, 24, Dictydium microcarpum 22. - V, EH.)

C. piriformis Schrader 3, 6. - V, St, EH, PS.

C. purpurea Schrader 3, 6, 13, - EH.

C. rufa (Roth) Rostaf. 13. - U.

(C. splendens (Schrader) Pers. 3, 6, 24. - V.

(C. tenella Schrader) 3, 6. - PS.

C. vulgaris Schrader 3 and 6 (as 'C. aurantiaca') 13, 22, 24. - V, U, St, EH, EP, PS. Note 8.

Diachea leucopodia (Bull.) Rostaf. 3, 6, 25. - V, U, St, EH, PS, KP.

Dictydium cancellatum $\rightarrow$ Cribraria cancellata

D. cernuum $\rightarrow$ Cribraria cancellata

D. microcarpum $\rightarrow$ Cribraria microcarpa

D. umbilicatum $\rightarrow$ Cribraria cencellata

Diderma chondrioderma (de Bary \& Rostaf.) G.Lister 6. - U. 
D. crustaceum Peck 6, 11 (as 'D. globosum'), 37. - V, $\mathrm{EK}, \mathrm{EH}, \mathrm{Ks}$.

D. effusum (Schweinitz) Morgan 13, 38. - U, Ks.

D. globosum Pers. 3, 6, 22 [35 is Diderma radiatum], 38. - EH, Ks. Note 9.

D. hemisphaericum (Bull.) Hornem 3, 6, 13, Chondrioderma michelii 24, Didymium hemisphaericum 22. - U, EH.

D. montanum (Meylan) Meylan 13, 38, - EP, Ks.

D. niveum (Rostaf.) Macbr. 13. - EK, PP.

D. radiatum (L.) Morgan 3, 6, 35 (as, 'D. globosum'), 38, Chondrioderma radiatum 24, Diderma stellare 22, D. umbilicatum 22, Perichaena phaeosperma 26, 29. - A, V, U, St, EH, EP, PS, Ks, InL. Note 10.

D. simplex (Schroeter) G. Lister. - EH. Note 11.

D. spumarioides (Fries) Fries 3, 6. - PP, PS.

D. stellare $\rightarrow$ D. radiatum

(D. testaceum (Schrader) Pers. 3, 6, Chondrioderma testaceum 24.)

D. umbilicatum $\rightarrow$ D. radiatum

Didymium anellus Morgan 13. - EH.

D. clavus (Albert. \& Schweinitz) Rabenh. 3, 4, 6, 24. - V, U, St, KP.

D. comatum (A. Lister) Nann.-Brem. 14. - EH, PH, PP.

D. complanatum $\rightarrow$ D. serpula

D. crustaceum Fries 3, 6, 24. - V, EK, EH, PS. Note 12.

D. crustaceum var. obducens $\rightarrow$ D. obducens

D. difforme (Pers.) S.F.Gray 3 (some of the specimens determined as 'Diderma globosum'), 6 (as in the preceding reference), 14, Chondrioderma difforme 23, 24. - V, U, St, EH, EP, PH, PS, PK, $\mathrm{KP}, \mathrm{Kn}, \mathrm{PP}$. Note 13.

D. dubium Rostaf. 14. - St, EH, PP.

D. effusum $\rightarrow$ D. squamulosum

D. farinaceum $\rightarrow$ D. melanospermum

D. hemisphaericum $\rightarrow$ Diderma hemisphaericum

D. iridis (Ditmar) Fries 14. - U, PP.

D. lobatum $\rightarrow$ D. melanospermum

D. melanospermum (Pers.) Macbr. 3, 5, 6, 16, 37, 38, D. farinaceum 22, 24, D. lobatum 22, D. physaroides 24. - V, U, EK, St, EH, ES, PH, PS, PP, $\mathrm{Ks}, \mathrm{InL}$.

D. melanospermum var. minus $\rightarrow$ D. minus

D. microcarpon $\rightarrow$ D. nigripes

D. minus (A.Lister) Morgan 38, D. melanospermum var. minus 6. - U, St, Ks.

D. nigripes (Link.) Fries 3, 6, 22, D. microcarpon 24. - V, U, EK, St, EH.

D. obducens Karsten, Not. Sällsk, Fauna Flora Fennica Förhandl. 9: 356 (1868) 3 (as 'D. squamu- losum'), 13, 22, D. crustaceum var. obducens 24. EH. Note 14.

D. physaroides $\rightarrow$ D. melanospermum

(D. serpula Fries 22, D. complanatum 3, 6, 24. - EH.)

D. squamulosum (Albert. \& Schweinitz) Fries 3, 6, 14, 16, 24, 38, D, effusum 24. - V, U, St, EP, PS, PK, PP, Ks, InL.

Echinostelium cribrarioides Alexop. 7. - U.

E. minutum de Bary 7, 9. - U, KmL, EnL.

Enerthenema papillatum (Pers.) Rostaf. 3, 6, 7, 23, 24, 37, 38. - V, U, EK, St, EH, PS, PK, Ks, InL.

Enteridium intermedium (Nann.-Brem.) Farr, Taxon 25: 514 (1976) 38, Reticularia intermedia 6. - Ks, InL. Note 15.

E. lycoperdon (Bull.) Farr, Taxon 25: 514 (1976). Reticularia lycoperdon 3, 6, 24, R. umbrina 22. A, V, U, EK, St, EH, PS, PK, PP, InL. Note 15.

E. olivaceum Ehrenb., Jahrb. Gewächsk. 1 (2): 57 (1819) 3, 6, 24, E. simillimum 23, E. simulans 24, Reticularia olivacea 22, R. versicolor 22. - V, St, EH, PS. Note 15. and 16.

E. simillimun $\rightarrow$ E. olivaceum

E. simulans $\rightarrow$ E. olivaceum

E. splendens (Morgan) Macbr. var. juranum (Meylan) Härkönen, Karstenia 19: 5 (1979) 13. - St, PP. Note 15.

Fuligo cinerea (Schweinitz) Morgan 13. - PP.

F. intermedia Macbr. 6. - U, PP.

F. muscorum Albert. \& Schweinitz 3, 4, 6, 37, 38, F. simulans 24, Reticularia muscorum 22. - V, U, EH, PH, PP, Ks. Note 17.

F. septica (L.) Wiggers 2, 3, 5, 6, 24, 37, 38, Aethalium septicum 18, 22, 32, A. septicum var. violaceum 19, 22, F. septica var. candida $3, \mathrm{~F}$. septica var. flava $3,4, F$. septica var. rufa $3, F$. septica var. violacea $3,4, F$. varians $23, F$. varians var. ecorticatum 23. - V, U, EK, St, EH, ES, EP, $\mathrm{PH}, \mathrm{PS}, \mathrm{PK}, \mathrm{KP}, \mathrm{PP}, \mathrm{Ks}$, InL.

F. septica var. candida $\rightarrow$ F. septica

F. septica var. flava $\rightarrow$ F. septica

F. septica var. rufa $\rightarrow$ F. septica

F. septica var. violacea $\rightarrow$ F. septica

F. simulans $\rightarrow$ F. muscorum

F. varians $\rightarrow$ F. septica

F. varians var. ecorticatum $\rightarrow$ F. septica

Hemiarcyria clavata $\rightarrow$ Hemitrichia clavata

H. karstenii $\rightarrow$ Hemitrichia karstenii

H. rubiformis $\rightarrow$ Metatrichia vesparium

Hemitrichia abietina (Wigand) G. Lister 3, 6, [36]. PS. Note 18.

H. clavata (Pers.) Rostaf. 3, 6, 37, 38, Hemiarcyria clavata 24, Trichia clavata 32. - EK, EH, PP, Ks.

H. karstenii (Rostaf.) A. Lister 3, 6, 24, Hemiarcyria 
karstenii 23. - V. Note 19.

(H. serpula (Scop.) Rostaf. 3, 6, 24.)

Hemitrichia vesparium $\rightarrow$ Metatrichia vesparium

Lachnobolus congestus $\rightarrow$ Arcyodes incarnata

Lamproderma arcyrioides (Sommerf.) Rostaf. 24. L. violaceum 3, 5, 6, 16, 24. - U, EH, PS, PP, InL.

L. arcyrionema Rostaf. 4 (as 'L. columbinum'), 6. PS. Note 20.

L. atrosporum Meylan 6, 37, 38. - ES, PP, Ks. EnL.

L. carestiae (Ces. \& de Not.) Meylan 6. - V, U.

L. columbinum (Pers.) Rostaf. 3, [4 is L. arcyrionema], 6, 23, 24, 38, L. physaroides 24, Stemonitis elegantula 22, S. physaroides 22. - V, U, EH, ES, KP, PP, Ks, InL. Note 21.

L. maculatum Kow., Mycologia 32: 654 (1970) 13. PP.

L. physaroides $\rightarrow$ L. columbinum

L. puncticulatum Härkönen, Karstenia 18: 20 (1978) 10. - U.

L. sauteri Rostaf. 13. - PS, U.

L. scintillans (Berk. \& Broome) Morgan 13. - U.

L. violaceum $\rightarrow$ L. arcyrioides

Leocarpus contextus $\rightarrow$ Physarum contextum

L. fragilis (Dickson) Rostaf. 1 (as 'Perichaena rostafinskii') 2, 3, 4, 5, 6, 9, 24, 37, 38, L. vernicosus 19, 22. - A, V, U, EK, St, EH, ES, EP, PH, PS, PP, Ks, KmL, EnL.

L. vernicosus $\rightarrow \mathrm{L}$. fragilis

Lepidoderma tigrinum (Schrader) Rostaf, 3, 6, 16, 24, 37, 38. - U, PS, Kn, PP, Ks, InL.

Licea flexuosa $\rightarrow$ L. variabilis

L. fragiformis $\rightarrow$ Tubifera ferruginosa

L. minima Fries 3, 6, 7, 9, 16, 21, 24, 32, Phelonites minima 22. - V, U, EH, St, KmL, EnL, InL.

L. variabilis Schrader, L. flexuosa 3, 4, 6, 23, 24. - V, EH, PS.

Lindbladia effusa $\rightarrow$ L. tubulina

L. tubulina Fries, L. effusa 3, 6. - V, U, EK, St, EH, PS, PK, PP.

Lycogala epidendrum (L.) Fries 2, 3, 4, 5, 6, 15, 16, $17,18,22,24,32,37,38$, L. miniata 39. - A, V, U, EK, St, EH, ES, EP, PH, PS, PK, KP, Kn, PP, $\mathrm{Ks}, \mathrm{KmL}, \mathrm{EnL}$, InL.

L. flavofuscum (Ehrenb.) Rostaf. 6, 33. - U, V.

L. miniata $\rightarrow$ L. epidendrum

Margarita metallica $\rightarrow$ Calomyxa metallica

Metatrichia vesparium (Batsch) Nann.-Brem., Hemiarcyria rubiformis 24, Hemitrichia vesparium 3, 6, Trichia pyriformis 20, 22, 24, 32. - V, U, EK, St, EH, PH, PS, PK, PP, InL.

Mucilago crustacea Wiggers, $M$. spongiosa 3, 4, 5, 6, 16, Spumaria alba $18,19,22,24,32,-A, V, U$, EK, St, EH, ES, PS, KP, PP, InL.

M. spongiosa $\rightarrow$ M. crustacea
Oligonema aeneum $\rightarrow$ Calomyxa metallica

Paradiacheopsis fimbriata (G.Lister \& Gran) J. Hertel 7, 9. - V, U, KmL, InL.

P. solitaria (Nann.-Brem.) Nann.-Brem. 7, 9. - U, $\mathrm{KmL}, \mathrm{EnL}$.

Perichaena chrysosperma (Currey) A.Lister 7, 9. - U, EnL.

P. corticalis (Batsch) Rostaf. 3, 4, 6, 9, 24, P. populina 17, 22, P. rostafinskii [1 is Leocarpus fragilis], 24. - V, U, EK, St, EH, EP, PS, EnL. Note 22.

P. phaeosperma $\rightarrow$ Diderma radiatum

P. populina $\rightarrow$ P. corticalis

$\mathrm{P}$. rostafinskii $\rightarrow \mathrm{P}$. corticalis

Phelonites minima $\rightarrow$ Licea minima

[Phelonites strobilina 22] Note 23.

Physarum albipes $\rightarrow \mathrm{P}$. robustum

P. apiculosporum Härkönen, Karstenia 18: 24 (1978) 12, 14. - Kn. Note 24.

$\mathrm{P}$. atrum $\rightarrow \mathrm{P}$. didermoides

P. auriscalpium Cooke 3, 6. - U, PS.

P. bivalve Pers. 38, Angioridium sinuosum 20, 22. Carcerina valvata 22, Physarum sinuosum 3, 4, 6 , 24. - V, U, St, EH, PH, PS, PP, Ks.

P. cinereum (Batsch) Pers. 3, 6, 7, 24, 38. - V, U, EK, St, EH, EP, KP, PP, Ks.

P. citrinum Schumacher 3, 6, P. schumacheri 24. - V.

P. compressum Albert. \& Schweinitz 6. - St.

P. confertum Macbr. 4 (as 'P. atrum'), 6 (some of the specimens as 'P. didermoides'). - U. Note 25.

(P. conglomeratum (Fries) Rostaf. 3, 6, 24.)

$\mathrm{P}$. connatum $\rightarrow \mathrm{P}$. notabile

P. contextum (Pers.) Pers. 3, 6, 24, Leocarpus contextus 22, Physarum contextum var. splendens 24. -A, U, EK, St, EH, PK, PP.

$\mathrm{P}$. contextum var. splendens $\rightarrow \mathrm{P}$. contextum

P. decipiens Curtis, Badhamia decipiens 3, 6, - PS.

P. diderma Rostaf. 6. - InL.

P. didermoides (Pers.) Rostaf. 6 (only one specimen), P. atrum [4 is P. confertum]. - V, U. Note 25.

P. globuliferum (Bull.) Pers. 3. - V.

P. lateritium (Berk. \& Rav.) Morgan 13, 38. - U, Ks.

P. leucophaeum Fries 23, 24, 38, P. nutans var. leucophaeum 3, 16. - V, EH, PS, Ks, InL.

(P. leucopus Link. 3, 6, 24.)

P. maydis $\rightarrow$ P. oblatum

P. melaleucum $\rightarrow$ Badhamia utricularis

P. notabile Macbr., P. connatum 3, 6. - EK, EH.

P. nudum Macbr. 9. - KmL, EnL.

P. nutans Pers. 3, 5, 6, 16, 18, 22, 32, 38, Tilmadoche gracilenta $24, \mathrm{~T}$. nutans $24, \mathrm{~T}$. nutans var. rigida 24. - A, V, U, EK, St, EH, ES, PK, PS, PP, Ks, InL. Note 26.

P. nutans var. leucophaeum $\rightarrow P$. leucophaeum 
P. oblatum Macbr. 7, 9, P. maydis 6. - U, St, EnL.

P. psittacinum Ditmar 5, 6, 34. - U.

P. pusillum (Berk. \& Curtis) G. Lister 7. - U.

(P. robustum (A. Lister) Nann.-Brem., P. albipes 22. - EH.)

P. rubiginosum Fries 3, 6, 24. - EH.

(P. schroeteri Rostaf., Śluzowce 419 (1875) 24. - EH.) Note 27.

P. schumacheri $\rightarrow$ P. citrinum

P. sinuosum $\rightarrow$ P. bivalve

P. straminipes A. Lister 3, 6. - U, EH.

P. thejoteum $\rightarrow$ P. virescens

P. utriculare $\rightarrow$ Badhamia utricularis

P. utriculare var. melaleucum $\rightarrow$ Badhamia utricula-

ris

P. virescens Ditmar 3, 4, 6, 23, 24, 37, 38, P. thejoteum 22. - V, U, St, EH, ES, Ks.

P. viride (Bull.) Pers. 3, 5, 6, 7, P. viride var. aurantium 3, P. viride var. incanum 3, Tilmadoche mutabilis 24. - A, V, U, St, EH, PS, PK, PP, InL.

$P$. viride var. aurantium $\rightarrow P$. viride

$P$. viride var. incanum $\rightarrow P$. viride

Reticularia atra $\rightarrow$ Amaurochaete atra

$\mathrm{R}$. intermedia $\rightarrow$ Enteridium intermedium

R. lycoperdon $\rightarrow$ Enteridium lycoperdon

R. muscorum $\rightarrow$ Fuligo muscorum

R. olivacea $\rightarrow$ Enteridium olivaceum

R. umbrina $\rightarrow$ Enteridium lycoperdon

R. versicolor $\rightarrow$ Enteridium olivaceum

Spumaria alba $\rightarrow$ Mucilago crustacea

Stemonitis axifera (Bull.) Macbr. 37, 38, S. ferruqinea 3, 6, 22, 24. - V, U, EK, St, EH, EP, PH, PS, PK, KP, Kn, PP, Ks.

$\mathrm{S}$. elegantula $\rightarrow$ Lamproderma columbinum

S. fasciculata $\rightarrow$ S. fusca

S. ferruginea $\rightarrow$ S. axifera

S. ferruginea var. smithii $\rightarrow$ S. smithii

S. flavogenita Jahn 3, 6, 37, 38. - V, U, St, PS, Ks, $\mathrm{KmL}$.

S. friesiana $\rightarrow$ Comatricha nigra

S. fusca Roth 2, 3, 4, 6, 18, 22, 24, 32, 38, Stemonitis fasciculata 39. - V, U, EK, St, EH, ES, EP, PH, PS, PK, PP, Ks, KmL, InL.

S. herbatica Peck 13. - U, EnL.

S. ovata $\rightarrow$ Comatricha nigra

S. pallida Wingate 7 . - U.

S. physaroides $\rightarrow$ Lamproderma columbinum

S. smithii Macbr., S. ferruginea var. smithii 3, 6. $\mathrm{V}, \mathrm{U}, \mathrm{EK}, \mathrm{EH}, \mathrm{PS}$.

(S. splendens Rostaf. 3, 6, 23, 24. - EH.)

S. splendens var. flaccida $\rightarrow$ Symphytocarpus flaccidus

S. typhoides $\rightarrow$ Stemonitopsis typhina
S. virginiensis Rex 13. - PK.

Stemonitopsis dictyospora (Celak.) Nann.-Brem. 13. $-V$, ES.

S. typhina (Wiggers) Nann.-Brem. 38, Comatricha typhina 24, C. typhoides 3, 6, 16, Stemonitis typhoides 22. - V, U, EK, St, EH, PS, PP, InL.

Symphytocarpus amaurochaetoides Nann.-Brem. 6 (as. 'Comatricha irregularis'), 13. - St. Note 28.

S. flaccidus (Morgan) Ing \& Nann.-Brem. 37, 38, Stemonitis splendens var. flaccida 3, 6. - U, EK, St, PS, Ks.

Tilmadoche gracilenta $\rightarrow$ Physarum nutans

T. mutabilis $\rightarrow$ Physarum viride

T. nutans $\rightarrow$ Physarum nutans

T. nutans var. rigida $\rightarrow$ Physarum nutans

Trichia affinis $\rightarrow T$. favoginea

T. anomala $\rightarrow$ Arcyodes incarnata

T. botrytis (J.F. Gmelin) Pers. 3, 5, 6, 7, 38, T. fragilis 24, T. purpurascens $24,31,32$. - A, V, U, PS, PP, Ks, EnL, InL. Note 29.

$\mathrm{T}$. botrytis var. lateritia $\rightarrow \mathrm{T}$. floriformis

T. chrysosperma $\rightarrow$ T. favoginea

T. clavata $\rightarrow$ Hemitrichia clavata

T. contorta (Ditmar) Rostaf. 3, 6, T. contorta var. inconspicua 3. - U, St, EH, EK, PS, PP, EnL.

$\mathrm{T}$. contorta var. inconspicua $\rightarrow \mathrm{T}$. contorta

T. decipiens (Pers.) Macbr. 2, 3, 4, 5, 6, 37, 38, Trichia fallax 18, 22, 24, T. pusilla 16 . - A, V, U, EK, St, EH, EP, PS, KP, PP, Ks, InL.

T. fallax $\rightarrow$ T. decipiens

T. favoginea (Batsch) Pers. 3, 5, 6, 38, T. affinis 3, 6, $\mathrm{T}$. chrysosperma [20 is T. varia], $22,24, \mathrm{~T}$. persimilis 3, 6, 22, 24, T. proximella 24. - V, U, St, $\mathrm{EH}, \mathrm{PH}, \mathrm{PS}, \mathrm{PP}, \mathrm{Ks}$, InL. Note 30. and $\mathbf{3 1}$.

T. floriformis (Schweinitz) G. Lister 6, T. botrytis var. lateritia 3. - A, EH.

$\mathrm{T}$. fragilis $\rightarrow \mathrm{T}$. botrytis

T. lutescens (A. Lister) A. Lister 6. - U, PP. InL.

$\mathrm{T}$. nigripes $\rightarrow \mathrm{T}$. varia

T. persimilis $\rightarrow$ T. favoginea

$\mathrm{T}$. proximella $\rightarrow \mathrm{T}$. favoginea

$T$. purpurascens $\rightarrow T$. botrytis

$\mathrm{T}$. pusilla $\rightarrow \mathrm{T}$. decipiens

T. pyriformis $\rightarrow$ Metatrichia vesparium

T. scabra Rostaf. 3, 4, 6, 16. - V, U, EK, EH, PS, PK, InL.

(T. subfusca $\operatorname{Rex} 3,6$. - PS.)

(T. turbinata $=$ 'a sessile Trichia' (Martin \& Alexopoulos 1969) 22, 24.)

T. varia (Pers.) Pers. 3, 6, 17, 20 (as 'T. chrysosperma') 22, 24, 32, 38, T. nigripes 22, T. varia var. olivacea 22. - A, V, U, EK, St, EH, EP, PS, Kn, $\mathrm{PP}, \mathrm{Ks}$, InL.

Tubifera ferruginosa (Batsch) J.F.Gmelin 3, 4, 5, 6, 
37,38 , Licea fragiformis $20,22,32$, Tubulina cylindrica 23, 24. - V, U, EK, St, EH, ES, PS, PK, $\mathrm{PP}, \mathrm{Ks}$.

Tubulina cylindrica $\rightarrow$ Tubifera ferruqinosa

\section{Notes}

Note 1. Trichia anomala Karsten, Not. Sällsk. Fauna Flora Fennica Förhandl. 9: 354 (1868) (Cornuvia anomala (Karsten) Karsten, Bidr. Känned. Finl. Nat. Folk 31: 131 (1879)), was mentioned by Martin \& Alexopoulos (1969) in a list of excluded and doubtful species. According to Lister (1925) it is a possible synonym of Trichia scabra. Hintikka (1920) refers to it as a synonym of Lachnobolus congestus (Sommerf.) Lister.

No type material of Trichia anomala (Type locality: Finland, Tavastia australis (EH): Forssa) was found, but one authentic specimen was available: Finland, Regio Aboënsis (V): Merimasku, ad lignum pini, 28.VI.1860 (?) Karsten 1451 (H). All that was left was a piece of membranous, ochraceous peridium and a yellow mass of spores and capillitium. This has all been made into a preparation in Hoyer's Medium. The capillitial threads are yellow, branching, densely warted, $3-4 \mu \mathrm{m}$ in diam. and so unlike those of Trichia or Cornuvia. The spores are light yellow, smooth, with some scattered warts, $6.5-7.6-8 \mu \mathrm{m}$ in diam. So Hintikka was probably right in placing the specimens in the synonymy of Lachnobolus congestus ( = Arcyodes incarnata).

Note 2. Arcyria obvelata (Oeder) Onsberg was recently introduced as the correct name for Arcyria (Trichia) nutans (Onsberg 1978).

Note 3. Lister (1925) records Arcyria stipata from Finland, but P.W.James (in litt.) announced that there is no Finnish material of the species in BM or K. So the record from Finland is possibly erroneous.

Note 4. The holotype of Badhamia lilacina (Fries) Rostaf. (Sweden: Femsjö, Herb. E.Fries) is in UPS and in good condition.

Note 5. Physarum utriculare (Bull.) Chev. var. melaleucum Nyl., Not. Sällsk. Fauna Flora Fennica Förhandl. 4: 126 (1859), also named P. melaleucum (Nyl.) Nyl. (Nylander \& Saelan 1859) and Badhamia utricularis var. melaleuca (Nyl.) Karst. (Karsten 1876), was later treated by Karsten (1879) as a synonym of Badhamia utricularis. All that is left of the original specimen of Nylander $(\mathrm{H})$ is a piece of wood, but there is a specimen collected by Karsten (No. 2091) in 1861 and identified as Badhamia utricularis f. melaleuca, which was possibly seen by Nylander himself. The specimen belongs to Badhamia utricularis, having the majority of the sporangia sessile, but also some with the typical strand-like stalks. So it is better to add this taxon to the synonym list of Badhamia utricularis rather than to that of $B$. capsulifera p.p., as mentioned in Martin \& Alexopoulos (1969:341).

Note 6. A well-preserved syntype specimen of Oligonema aeneum Karsten, Bidr. Känned. Finl. Nat. Folk 31: 131 (1879) exists in H: Fennia, Tavastia australis (EH): Tammela, Mustiala, ad lignum pini, IX.1870 Karsten 3173. It consists of clustered sessile sporangia and plasmodiocarps of Calomyxa metallica.

Note 7. Comatricha ellae was first described as $C$. nannengae (Härkönen 1977b), but the name proved to be a later homonym (see Härkönen 1978c). More pictures of the species were published by Mitchell (1978). It is now known from Norway, Finland and Great Britain.

Note 8. All the specimens of Cribraria aurantiaca Schrader in the Finnish herbaria (H, HFR, OULU, TUR) belong to $C$. vulgaris Schrader (see Härkönen 1979).

Note 9. Some of the specimens quoted in references 3,6 and 22 are wrongly determined, belonging to $D$. crustaceum and $D$. difforme.

Note 10. The Finnish type material of Perichaena phaeosperma Karsten, Rev. Mycol. 9: 11 (1887), labelled: Tavastia australis (EH): Tammela, Mustiala, in ligno populi, IX.1886 Karsten 2092 (H), is quite typical Diderma radiatum.

Note 11. Diderma simplex (Schroeter) G.Lister

EH: Hauho, Hakkila, on a drained pine bog along a ditch, on grasses, VIII.1977 Alanko $33661(\mathrm{H})$. According to Martin \& Alexopoulos (1969), a widely distributed, but rare species. Reported from Denmark (Bjørnekaer \& Klinge 1964). New to Finland.

Sporangia sessile, crowded, $0.5-0.7 \mathrm{~mm}$ in diam., orange-ochre, (according to Maerz \& Paul (1950), Cinnamon 12 E 7). Peridium single, rugulose. Columella inconspicuous. Capillitium hyaline. Spores pale lilaceous grey in transmitted light, very minutely spinulose with clusters of larger spines, 8.5 $-9.5-10 \mu \mathrm{m}$ in diam.

Note 12. A well-preserved type specimen of Didymium crustaceum: Russia, Petropoli (= Leningrad), coll. Weinmann, det. E.Fries, deposited in UPS clearly belongs to the present species. The type locality is not 'the Alps' as erroneously mentioned in Martin \& Alexopoulos (1969). Fries 
(1829) reported $D$. crustaceum from the Alps, but the only specimen he examined was that from Russia. Fries cited Spumaria physaroides Pers., but Farr (1976b) placed it in the synonymy of Didymium melanospermum (Pers.) Macbr.

Note 13. There have been difficulties in identifying Didymium difforme, because the crystalline structure of the outer peridium is sometimes hard to discern (see Härkönen \& Koponen 1978). The species is common in Finland.

Note 14. Didymium obducens Karsten has been shown to be an earlier name for $D$. fulvum Sturgis (see Härkönen 1979). Holotype: Finland. Tavastia australis (EH): Tammela, Mustiala, Myllyperä, at base of Alnus incana, X.1866 Karsten 2035 (H).

Note 15. Farr (1976a) pointed out that the generic name Reticularia Bull. is a later homonym of the lichen genus Reticularia Baumg. and must be replaced with Enteridium Ehrenb.

Note 16. Karsten (1876) used the name 'Enteridium simillimum Rostaf.', but that name was not published by Rostafiński. Karsten probably made a mistake and meant Enteridium simulans Rostaf. (= E. olivaceum). The specimen to which Karsten referred is deposited in $\mathrm{H}$ : Finland: Tavastia australis (EH): Tammela, Pähk(i)järvi, ad Alnum incan., 20.IX.1867 Karsten 2098. Inside the envelope is a piece of paper, where Karsten has written both $E$. simulans and $E$. simillimum.

Note 17. Finnish specimens of Fuligo simulans Karsten, Bidr. Känned. Finl. Nat. Folk 31: 108 (1879) exist in $\mathrm{H}$ : Tavastia australis (EH): Tammela, Mustiala (Myllyperä) 8.IX.1871 Karsten 2693 and 9,IX.1871 Karsten 2094. This is most probably the original material mentioned in the description of the species. The specimens clearly belong to $F$. muscorum.

Note 18. The specimen mentioned by Roivainen (1964) has been dried before it matured, so that it is impossible to determine, but it is probably not Hemitrichia abietina.

Note 19. Hemitrichia karstenii (Rostaf.) A.Lister is based on Hemiarcyria karstenii Rostaf., Śluzowce Dod. 41 (1876), which was based on two syntypes, one from Finland, the other from Ceylon. As mentioned in Martin \& Alexopoulos (1969), Karsten's specimen should be considered the (lecto)type, although Lister (1925) referred to the Ceylon collection as the type. There is a well-preserved isosyntype from Finland: Ab (V): Turku, Runsala
(Ruissalo), ad lign. acerin,, 13.IX.1866 Karsten 2095 (H). It consists chiefly of long, (up to $6 \mathrm{~mm}$ ), branching plasmodiocarps. The peridium is thick, and dark brown. The capillitial threads are long, sparsely branching, with hardly any expansions, 3-4 $\mu \mathrm{m}$ broad, the spirals very faint. The spores are light yellow, minutely warted, $10-11-11.5 \mu \mathrm{m}$ in diam.

Ing (1965) proposed that the species should be included in Trichia contorta (Ditmar) Rostaf., as var. karstenii (Rostaf.) Ing, because apart from the branching elaters and a tendency to form plasmodiocarps, there is no character which separates $H$. karstenii from $T$. contorta. This also applies to the Finnish material of $T$. contorta, but Ing's treatment is not followed here, because the whole genus Hemitrichia is distinguished from Trichia by the type of capillitium. According to Ing (1965) Hemitrichia is not a 'satisfactory genus', but he made no proposal regarding the other members of Hemitrichia, and himself listed several Hemitrichia species as late as 1976 (Ing 1976). Until the whole species complex Trichia - Hemitrichia has been revised, I find it better to recognize Hemitichia karstenii.

Note 20. As pointed out by Martin \& Alexopoulos (1969), No. 8 in Hintikka's exsiccati (labelled 'Lamproderma columbinum') is Lamproderma arcyrionema.

Note 21. No material was found for Stemonitis elegantula Karsten, Not. Sällsk. Fauna Flora Fennica Förhandl. 9: 354 (1868). Later Karsten (1879) himself added the name to the synonym list of Lamproderma columbinum.

Note 22. No type material could be traced for Perichaena rostafinskii Karsten, Bidr. Känned. Finl. Nat. Folk 31: 130 (1879), described from Finland, St: Tyrvis (Tyrvää). The name is commonly regarded as a synonym of $P$. corticalis (Martin \& Alexopoulos 1969).

Note 23. Karsten (1868) listed 'Phelonites strobilina (A. S.)' as a myxomycete, but later (1871) treated the species as a rust. Liro (1908) considered Phelonites strobilina Karsten a synonym of Pucciniastrum padi (Kunze \& Schm.) Dietel ( $=P$. areolatum (Fries) Otth.).

Note 24. The holotype of Physarum apiculosporum (Härkönen 1335) is in $\mathrm{H}$, the isotype in herb. N.E.Nannenga-Bremekamp and in the University of California, Berkeley, were it has been cultivated from spore to spore in a halfstrength corn meal agar (D. Betterley, in litt.). 
Note 25. Physarum atrum auct. non Schweinitz has been figured, e.g. by Lister (1911), as a small, dark, heaped species. T.J.Hintikka (1924) distributed this species most probably identified according to Lister, as No. 14 in his exsiccati. Macbride (1922) showed that the type specimen of $P$. atrum Schweinitz is a limeless $P$. didermoides (Pers.) Rostaf. So $P$. atrum Schweinitz had to be added to the list of synonyms of $P$. didermoides. For $P$. atrum auct. non Schweinitz, Macbride (1922) established a nomen novum, $P$. confertum Macbr. The specimen in Hintikka's exsiccati is also $P$. confertum.

Note 26. Tilmadoche nutans (Pers.) Rostaf. var. rigida Rostaf., Śluzowce 128 (1874) has not been referred to in any major monographs, e.g. those of Lister (1925), Krzemieniewska (1960), Martin \& Alexopoulos (1969), or Nannenga-Bremekamp (1974). Karsten (1879) writes 'cum forma genuina', which indicates that this variant probably does not deserve recognition. No corresponding specimen of Karsten has been found.

Note 27. Karsten (1879) recorded Physarum schroeteri Rostaf. from Tammela, but no specimen was found. Lister (1925) cited the name as a possible synonym for $P$. citrinum Schumacher. Krzemieniewska (1960) treated $P$. schroeter $i$ as valid, and Martin \& Alexopoulos (1969) suggested that she might be right. According to the description by Rostafiński (in Polish), P. schroeteri seems to have rather distinctive properties, e.g. compressed sporangia; a dehiscing peridium that leaves a collar round the stalk; the stalk calcareous inside and shiny as if lacquered outside; the lime granules small, rounded, regular, located in the capillitium like beads in a rosary.

Note 28. The Finnish specimens determined as Comatricha irregularis Rex (Härkönen 1974) have been re-examined and shown to be Symphytocarpus amaurochaetoides (Härkönen 1979).

Note 29. Trichia purpurascens Nyl., Not. Sällsk. Fauna Flora Fennica Förhandl. 4: 126 (1859). The type specimen from Finland: U: 'ad Helsingfors' (= Helsinki), oct. $1858 \mathrm{~W}$. Nylander $(\mathrm{H})$ is quite typical Trichia botrytis.

Note 30. Trichia persimilis Karsten, Not. Sällsk. Fauna Flora Fennica Förhandl. 9: 353 (1868). In several specimens of Karsten, e.g. the holotype from Finland: Tavastia australis (EH): Tammela, Mustiala, 2.IX.1867 Karsten $2096(\mathrm{H})$, the spores have a broken reticulation and interrupted border. This distinguishes the specimens from the Finnish material of Trichia favoginea (Batsch) Pers. s.str., which has banded-reticulated spores. Farr (1958), however, showed convincingly, that complete intergradation exists in every respect between $T$. favoginea and $T$. persimilis. Her treatment is followed here.

Note 31. Trichia proximella Karsten, Bidr. Känned. Finl. Nat. Folk 31: 139 (1879) is commonly treated as a synonym of $T$. favoginea (Batsch) Pers. (Martin \& Alexopoulos 1969). Only one syntype specimen was detected: Fennia, Regio aboënsis (V): Merimasku, Kaita, ad quercum (vel tiliam?), VII.1860 Karsten $1450(\mathrm{H})$. It clearly belongs to $T$. favoginea.

\section{References}

The publications with a serial number contain chorological information about Finnish Myxomycetes and are referred to in the check-list.

1 Anonymous 1903: Eräs limasieni herättänyt huomiota. - Luonnon Ystävä 7: 224.

Bjørnekaer, K. \& Klinge, A. 1963: Die dänischen Schleimpilze. Myxomycetes Daniae. - Friesia 7: 149-296.

Ehrenberg, C. 1819: Fungorum nova genera tria. Jahrb. Gewächsk. 1 (2): 51-58.

2 Eklund, O. 1943: Zur Pilzflora des Schärenarchipels SW-Finnlands. - Mem. Soc. Fauna Flora Fennica 18: $2-21$.

Farr, M. 1958: Taxonomic studies in the Myxomycetes 1. The Trichia favoginea complex. - Mycologia 50: $357-369$.

-"- 1976a: Reticularia Baumgarten (lichens) versus Reticularia Bulliard (Myxomycetes). - Taxon 25: 514.

_"- 1976b: Flora Neotropica 16. Myxomycetes. - 304 pp. New York.

Forsman, A. 1898: Tarullisia tutkimusaiheita kotimaisen kasvistomme ja kasvinimistömme alalta. Suomen Museo 5: 71-100.

Fries, E. 1829: Systema mycologicum ... 3. - 202 pp. Gryphiswaldiae.

-"- 1880: Kritisk ordbok öfver svenska växtnamnen. 177 pp. Stockholm.

Hintikka, T. 1914: Limasieniä keräämään! - Luonnon Ystävä 18: $11-16$.

3 -"- 1920 ('1919'): Révision des myxogastres de Finlande. - Acta Soc. Fauna Flora Fennica 46 (9): $1-43$.

4 -". 1924: Myxogastres fennici exsiccati. Dec. 1-2 (No. $1-20)$, index.

_'- 1931: Sienimaantieteellisestä (mykogeograafisesta) tutkimustyöstä, varsinkin Suomessa. - $49 \mathrm{pp}$. Helsinki.

-"- 1935: P.A. Karsten. Anlässlich seines 100. Geburtstages 16. Februar 1934. - Friesia 1: 203-229.

-'-1950: P.A. Karstenin elämäntyöstä. Karstenia 1: 5-14.

5 Hintikka, V. 1963: Notes on Finnish Myxomycetes. Karstenia 6-7: 110 . 
6 Härkönen, M. 1974: Über die finnischen Schleimpilzen. - Karstenia 14: 54-81.

"- 1975: Limasienet, Myxomycetes. - Sienilehti 27: 62-67.

7 -"- 1977a: Corticolous Myxomycetes in three different habitats in southern Finland. - Karstenia 17: 19-32.

8 -"- 1977b: Comatricha nannengae, a new species of Myxomycetes. - Karstenia 17: 87-89.

9 -'- 1978a: On corticolous Myxomycetes in northern Finland and Norway. - Ann. Bot. Fennici 15: $32-37$.

10 -"'-1978b: Lamproderma puncticulatum, a new species of Myxomycetes. - Karstenia 18: 20-22.

11 -"-1978c: Comatricha ellae, nomen novum (Myxomycetes). - Karstenia 18: 23.

12 -'- 1978d: A new species of Myxomycetes, Physarum apiculosporum, described and cultivated. Karstenia 18: 24-26.

13 -"- 1979: Additions and corrections to the Finnish flora of Myxomycetes. - Karstenia 19: 1-7.

14 Härkönen, M. \& Koponen, H. 1978: Myxomycetes developed on grain in moist chamber cultures. Karstenia 18: $58-62$.

Ing, B. 1965: Notes on Myxomycetes. - Trans. Brit. Mycol. Soc. 48: 647-651

-"- 1976: The natural history of Slapton Ley Nature Reserve 11. Myxomycetes (slime moulds). - Field Stud. 4: $441-456$.

Jenssen-Tusch, H. 1867: Nordiske plantenavne. - 358 pp. Kjøbenhavn.

15 Kallio, P. 1969: Turun sieninäyttely. - Sienitietoja 1969 (4): 6-7.

16 Kallio, P. \& Kankainen, E. 1966: Additions to the mycoflora of northernmost Finnish Lapland. Ann. Univ. Turku. (A 2) 36 (Rep. Kevo Subarctic Sta. 3): 177-210.

17 Karsten, P. 1865: Fungi Fenniae exsiccati. Cent. 1-2 (No. 101-300). - Ảbo.

18 -"-1866a: Enumeratio Fungorum et Myxomycetum in Lapponia orientali aestate 1861 lectorum. - Not. Sällsk. Fauna Flora Fennica Förhandl. 8 (ny ser. 5): $193-224$

19 -"- 1866b: Fungi Fenniae exsiccati. Cent. 4-6 (No. 301-600). - Åbo.

20 -"- 1867: Fungi Fenniae exsiccati. Cent. 7 (No. 601-700) - Åbo.

21 -"'-1868a: Fungi Fenniae exsiccati. Cent. 8 (No. 701-800). - А̊bo.

22 -"- 1868b: Gastero- et Myxomycetes circa Mustiala crescentes. - Not. Sällsk. Fauna Flora Fennica Förhandl. 9 (ny ser. 6): 349-356.

-"-1971: Peronosporei, Aecidiei et Ustilaginei, e regione Mustialaënsi hucusqve cogniti. - Not. Sällsk. Fauna Flora Fennica Förhandl. 11 (ny ser. 8): $262-268$

23 -"- 1876: Symbolae ad Mycologiam Fennicam 3. Medd. Soc. Fauna Flora Fennica. 1: 55-59.

24 -"- 1879: Mycologia Fennica 4. - Bidrag Känned. Finlands Natur Folk 31: 89-142.

25 -"- 1884: Fungi rariores Fennici atqve nonnulli Sibirici a D:re Edv. Vaino lecti. - Medd. Soc. Fauna Flora Fennica 11: 136-144.

26 -'- 1887a: Symbolae ad Mycologiam Fennicam 18. Medd. Soc. Fauna Flora Fennica 14: 78-84.

27 -"- 1887b: Symbolae ad Mycologiam Fennicam 19. Medd. Soc. Fauna Flora Fennica 14: 85-94.

28 -"- 1887c: Symbolae ad Mycologiam Fennicam 20. Medd. Soc. Fauna Flora Fennica 14: 95-102.

29 -"- 1887d: Fungi novi vel minus bene cogniti Fenniae et Galliae. - Rev. Mycol. 9: 9-11.
Kotilainen,M. 1954: Toivo Juho Hintikka. - Mem Soc. Fauna Flora Fennica 29: 118-124.

Kowalski, D. 1970: The species of Lamproderma. Mycologia 62: $621-672$.

Krzemieniewska, H. 1960: Śluzowce. - 313 pp Warszawa.

Kujala, V. 1953: T.J.Hintikka 8.2.1888-20.6.1952. Karstenia 2: 5-8.

"- 1954: Dr. T.J.Hintikka 8. Februar 1888 - 20 Juni 1952. - Friesia 5: 1-5.

Kukkonen, I. \& Ahti, T. 1978: Accessions to the Botanical Museum, University of Helsinki/Helsingfors in 1977-1978. - Mem. Soc. Fauna Flora Fennica 54: $75-78$.

Liro, J. 1908: Uredineae Fennicae, Finlands rostsvampar. - Bidr. Känned. Finlands Natur Folk 65: $1-642$.

Lister, A. 1911: A monograph of the Mycetozoa. 2nd ed., revised by G. Lister - 302 pp., 200 pls. London.

-'-1925: A monograph of the Mycetozoa. 3rd ed., revised by G. Lister. - 296 pp., 222 pls. London.

Macbride, T. 1922: The North American slime-moulds. 2nd ed. - 299 pp., 23 pls. New York.

Maerz, A. \& Paul, M. 1950: Dictionary of color. 2nd ed. -208 pp. Iowa City.

30 Marklund, G. 1954: Helsingin Yliopiston kotimaisten kasvitieteellisten kokoelmien kasvu v. 1953-1954. - Mem. Soc. Fauna Flora Fennica 30: 109-112.

Martin, G. \& Alexopoulos, C. 1969: The Myxomycetes. -560 pp. Iowa City.

Mela, A. 1892: Kasvioppi Suomen kouluille. 3rd ed. 460 pp. Helsinki.

Mitchell, D. 1978: A key to the corticolous Myxomycetes 2. - Bull. Brit. Mycol. Soc. 12: 90-107.

Mossberg, B., Nilsson, S. \& Persson, O. 1977: Sienet luonnossa 1. (Finnish ed. T. Ulvinen). $-128 \mathrm{pp}$. Helsinki.

Mäkelä, K. 1972: Seed borne fungi on cultivated grasses in Finland. - Acta Agr. Fennica 124 (2): 1-44.

Nannenga-Bremekamp, N. 1974: De nederlandse myxomyceten. - 440 pp. Zutphen.

Norrlin, J. 1899: William Nylander. — Luonnon Ystävä 3: $71-83$.

31 Nylander, W. 1859: Analyses mycologicae. - Not. Sällsk. Fauna Flora Fennica Förhandl. 4 (ny ser. 1): $119-126$.

-"-1861: [Annotations] In: Fries, E.: Note sur la distribution géographique des champignons. Ann. Sci Nat. (4 Bot.) 15: 10-35.

32 Nylander, W. \& Saelan T. 1859: Herbarium musei fennici. Förteckning öfver finska musei växtsamling. $-118 \mathrm{pp}$. Helsingfors.

Onsberg, P. 1978: Notes on danish Myxomycetes 2. Arcyria obvelata: a new name for Arcyria (Trichia) nutans. - Mycologia 70: 1284-1286.

Reinholm, H. 1851: Suomalaisia kasvu-nimejä. Suomi 10: 159-301.

33 Roivainen, H. 1961: Helsingin Yliopiston Kasvimuseon itäfennoskandisten itiökasvikokoelmien kasvu 1959-1960. - Mem. Soc. Fauna Flora Fennica 36: $136-137$.

34 -"-1962: Helsingin Yliopiston Kasvimuseon itäfennoskandisten itiökasvikokoelmien kasvu 1960-1961. - Mem. Soc. Fauna Flora Fennica 37: $286-288$

35 -'”- 1963: Helsingin Yliopiston Kasvimuseon itäfennoskandisten itiökasvikokoelmien kasvu 1962 - 1963. - Mem. Soc. Fauna Flora Fennica. 39: $190-192$. 
36 Roivainen, H. 1964: Helsingin Yliopiston Kasvimuseon itäfennoskandisten itiökasvikokoelmien kasvu 1963-1964. - Mem. Soc. Fauna Flora Fennica 40: $221-223$.

Rostafiński, J. 1874-1875: Sluzowce (Mycetozoa), monografia. - Pamiętnik Towarzystwa Nauk Ścislych (Paryż) 5: $1-432,1-13$ pls.

-'- 1976: Dodatek 1 do monografii śluzowców. Pamiętnik Towarzystwa Nauk Ścislych (Paryż) 8: 43.

Suhonen, P. 1936: Suomalaiset kasvinnimet (German ref.: Finnische Pflanzennamen.) - Ann. Bot. Soc. Vanamo 7 (1): 1-465.

Suomalainen, P. 1948: T.J. Hintikka 60-vuotias. Luonnon Tutkija 52: 16-17.
Turun Suomalaisen Yliopiston Vuosikirja 1925. - 89 pp. Turku.

Ulvinen, T. 1978: Pohjolan siententutkijat Kuusamossa. - Sienilehti 30: 59-62.

37 Ulvinen, T., Ohenoja, E., Ahti, T. \& Alanko, P. 1978: A preliminary check-list of the fungi (incl. lichens) of the Kuusamo biogeographical province, NE Finland. - Oulun Yliopiston Oulangan Biol. Aseman Monisteita 1: 1-57.

38 -"-1979: A check-list of fungi (incl. lichens) of the Kuusamo biogeographical province, NE Finland. Oulanka Rep. (in preparation).

39 Wahlenberg, (i. 1812: Flora Lapponica. - 550 pp., 30) pls. Berolini.

Accepted for publication

on February 13, 1979 